\title{
Bilderbogen und ihre Benutzer - Schlachtenbilder und Heilige im Spiegel zweier französischer Autoren
}

\section{Katharina Siefert}

Die Fragen nach der Rezeption von Bilderbogen und nach der Art ihrer Aufbewahrung oder Verwendung lassen sich nur anhand einiger weniger Memoiren von Autoren aus dem 19. Jahrhundert beantworten. Diese wurden in der Bilderbogenforschung häufig zitiert und sind hinlänglich bekannt. Insbesondere INGEBORG WEBER-KELLERMANN hat in ihrem Aufsatz „Bilderbogen im Kinderzimmer“ einige dieser Autoren zitiert, in deren Jugenderinnerungen Bilderbogen Erwähnung finden ${ }^{1}$. Die Spanne reicht von KARL AUGUST VARNHAGEN von ENSEs Erinnerungen aus der Zeit vor 1800 bis hin zu EMIL NOLDEs Beschreibung einer Lektion in hoher und niederer Kunst - zu Letzterer wurden die Bilderbogen gezählt. Ebenso fehlen auch nicht die schwärmerischen Eindrücke LUDWIG RICHTERs. Alle Autoren nennen meist Bilderbogen deutscher Verlage, häufig aus Berlin oder Neuruppin. Erinnert sei auch an THEODOR FONTANEs Bemerkungen zu Bilderbogen der Firma GuSTAV KÜHN in Neuruppin.

Bei der Bewertung solcher Äußerungen in Autobiografien ist zu prüfen, mit welcher Intention diese verfaßt wurde. Zwar handelt es sich um Zeitzeugnisse, vor allem jedoch um Selbsterkundungen: Der Autor notiert das, was ihm erinnerungswürdig erscheint. Der zeitliche und intellektuelle Abstand zu einem Ereignis führt zwangsläufig zu einer Auswahl des Erinnerten wie auch zu einer nachträglichen Sinngebung, häufig auch zur Aufbesserung der Biografie. Solchermaßen halten die Texte Informationen über das psychische Erleben ihrer Akteure bereit. Die narrative Identität eines Autors muß daher beachtet werden, um das Geschriebene auf eine objektive Darstellung eines Sachverhaltes oder Gegenstandes zu bewerten.

Im Falle der Bilderbogen handelt es sich bei den o. g. Autoren meist um abwertende Äußerungen: Bilderbogen werden als Surrogate von bildender Kunst und Geschichte für den einfachen, ,unverbildeten“ Geschmack bewertet. In diesem Sinne werden sie sowohl als volkstümlich als auch kindgerecht betrachtet. So erklärt sich, weshalb in autobiografischen Erinnerungen an die Kinder- und Jugendzeit Bilderbogen häufig Erwähnung finden, wie auch in den beiden nachfolgenden Beispielen.

Die beiden Texte aus dem französischen Sprachraum können hier in deutscher Übersetzung hinsichtlich Wirkungsgeschichte und Rezeption von Bilderbogen erstmals vorgestellt werden.

In seinem autobiographischen Roman „Die Linden von Lautenbach. Eine deutschfranzösische Lebensgeschichte“ beschreibt der Elsässer JEAN EGEN, geboren 1920, aus der Perspektive eines Kindes, das nicht weiß, welcher Nation es sich zugehörig fühlen soll, die wechselvolle Geschichte seiner Familie aus der Provinz zwischen Frankreich

In: Volkskunst. Zeitschrift für volkstümliche Sachkultur 7 (1984) H. 2, S. 5-10. 
und Deutschland ${ }^{2}$. So wird der patriotische Eifer des Knaben Seppala [es handelt sich um den Vater des Autors, d. V.] von den Bilderbogen aus dem Besitz des Großvaters beeinflußt:

Und da ist nicht nur mein Großvater, der erzählend das Napi-Epes [i. e. Napoleon-Epos, d. V.] wiederaufleben läßt, da ist auch ein Bilderschatz, der sorgsam in einer Blechschachtel gänz unten im Schrank verwahrt wird und der sich abends vor dem faszinierten Seppala ausbreitet. Da sind Bilder und Worte, die Belagerung von Toulon, die Brücke von Arcolo, die Sonne über der Schlacht von Austerlitz. ... Andere Bilder treiben die Tränen in Seppalas Augen, Waterloo, am Abend nach der Schlacht, oder wie Napi auf dem Fels seiner Exil-Insel St. Helena sitzt und nostalgisch in die Ferne blickt ${ }^{3}$.

Offensichtlich hat der Großvater, um seine Erzählungen über die Schlachten Napoleons eindringlich zu gestalten, die anschaulichen Bilderbogen zu Hilfe genommen. Sowohl französische Geschichte als auch Bildwissen werden durch die genannten Bogen vermittelt. Das visuelle Medium hat denn auch den Knaben nachhaltig beeinflußt. Diese Bilderbogen erscheinen ihm als sehr wertvolles Gut, das in einer bestimmten Weise sicher verwahrt und anscheinend nur unter der Aufsicht eines Erwachsenen hervorgeholt und ehrfürchtig betrachtet wurde.

Vermutlich handelt es sich bei den von JEAN EGEN genannten Bilderbogen um Blätter des Verlages PELLERIN aus Épinal, der eine weit verbreitete Serie mit Darstellungen aus dem Leben NAPOLEONs herausgegeben hatte. Diese schablonenkolorierten Holzschnitte nach den Entwürfen von FRANÇOIS GEORGIN wurden zwischen 1820 und 1835 verlegt ${ }^{4}$. Zumindest bei einem Bogen, nämlich der „Schlacht bei den Pyramiden“ besteht Gewißheit, daß der Bilderbogen in der Épinaler Serie „Bataille des Pyramides“ produziert worden war, denn Egen zitiert aus der Bildlegende den berühmten Ausspruch Napoleons: „Soldaten, vom Gipfel dieser Pyramiden sehen vierzig Jahrhunderte auf euch herab"

EGEN beschreibt noch weitere Bilderbogen, betrachtet an einem ungewöhnlichen Ort der Reflexion:

Ganz hinten im Garten ist das Klo, eine einfache Bretterbude, aber darin hängen bunte Bilderbögen mit dem Leben der heiligen Johanna von d'Arc. ... Die Ausschmückung dieser stillen, für die Betrachtung sehr geeigneten Stätte dient der Erhebung der Seele. Wenn ein Sonnenstrahl durch das kleine in die Brettertür geschnittene Herz dringt und das Refugium in Licht taucht, dann vertieft sich Joseph in die Bilder. Auf dem rustikalen Bretterthron sitzend, lebt er das Leben der Heldin nach. Ruhe und Abgeschiedenheit von der Welt bringen ihn sogar dazu, die Rolle der kleinen Hirtin von Domrémy selbst zu übernehmen ${ }^{6}$.

Heiligenbilderbogen verwandeln diesen sehr profanen Ort in einen geradezu mystischen Erfahrungsraum. Ironisch beschreibt JEAN EGEN die kindlichen Phantasien des

2 EGEN, JEAN: Die Linden von Lautenbach. Eine deutsch-französische Lebensgeschichte. Hamburg 1986.

Ebd., S. 64.

MARTIN, DENIS: Images d'Épinal. Quebec 1995, S. 214f.

EGEN (wie Anm. 2), S. 64.

Ebd., S. 64f. 
Knaben, die sich geschlechtsspezifisch korrekt an Schlachtenbilder entzünden. Hierzu gehören auch die Bilderbogen mit der Geschichte JEANNE D'ARCs, handelt es sich letztlich doch um eine Kämpferin für den christlichen Glauben.

Für die Rezeptionsgeschichte der genannten Bilderbogen ist es bedeutsam, daß es sich bei dem Knaben Seppala nicht um den Autor selbst handelt, sondern um dessen Vater. So berichtete JEAN EGEN einerseits aus der Perspektive Seppalas, andererseits beschreibt er die genannten Bilderbogen offenbar aus eigener Anschauung. Sie dienen im Roman als Medium, die widersprüchlichen Gefühle hinsichtlich der Nationalität, das Elsaß war zu diesem Zeitpunkt deutsches „Reichsland“ geworden, zu verdeutlichen. Bezeichnend für die Einschätzung und Bewertung der Bilderbogen ist, daß sie nur als Kindheitserinnerungen des Vaters manifest sind, im weiteren Leben des Autors dagegen haben sie keinen Platz mehr und finden daher im Roman keine weitere Erwähnung.

Heiligendarstellungen und Bilderbogen anderer Art sind es, die HUGUETTE DREIKAUS als Mädchen in einem elsässischen Dorf in den 50er Jahren vor Augen hat. In ihren Buch „Das Elsaß, das ich meine. Kleine Philosophie aus der Provinz“ schildert die Schauspielerin und Kabarettistin mit liebevoller Ironie die elsässische Alltagskultur. So erläutert sie dem Kapitel „Gemälde und Gesangbuchbildchen“ den Gebrauch und den Stellenwert religiöser Bilderbogen:

Über meinem Bett weinte Jesus in sämtlichen denkbaren Blautönen über das verlorene Jerusalem. In unserer Stubb führte ein in rosa Musselin gewandeter Engel zwei Kinder durch die reißenden Wasser eines Wildbaches. Im Alkoven meiner Großmutter vergoß eine hohlwangige Mater dolorosa bittere Tränen. Auf dem Nachttisch meiner Eltern wurde das Herzjesu in gelben Flammen verzehrt. In unseren elsässischen Häusern gab es fromme Bilder zu Hauf ... sie gehörten zu unserem Alltag. Jeden Tag wurde einem jeden dieser Bilder ein Gebet entgegengeschickt. ... Wo die Bilder herkamen wußte kein Mensch. ... Die Christusdarstellungen waren so, wie man sich Christusse vorstellte: mager, mit langen Haar, bärtig, von unsagbarer Traurigkeit. Welche Schule? Man brauchte doch nicht zu wissen wer sie gemalt hatte. Hauptsache sie waren da, denn mit ihnen hatte man alle mögliche Hilfe in greifbarer Nähe. ... Jedes von Ihnen war eine Art Zauberpforte zum Glück und verschaffte uns Zugang zu haufenweise Wohltaten und Ablässen. ... Zum Glück hatte ich meine Ikonen, meine Heljele [Heiligenbildchen]: Erinnerungen an Taufen mit erbaulichen Sprüchen und Lehrsätzen fürs Leben, bunte Lithographien, die einst zur Kommunion geschenkt wurden ... Zu jedem Ereignis gehörte ein Bild. Und jedes Bild hatte einen festen Platz im Haus ${ }^{7}$.

Diese Textstelle verdeutlicht anschaulich die Art der „Benutzung“ der verschiedenen Bilderbogen und rechtfertigt die Länge des Zitats. Bemerkenswert ist der feste Platz eines jeden Bilderbogens. Heiligenbilder, insbesondere von Christus und der Mutter Gottes, finden sich in den Schlafzimmern. Durch das abendliche Gebet zu den Heiligen konnte das Gefühl der Hilflosigkeit, während der Nacht schlafend einer Gefahr ausgesetzt zu sein, gebannt werden. Die Abbildungen der Heiligen erhalten auf diese Weise geradezu apotropäische Funktion. HUGUETTE DREIKAUS selbst spricht von

DREIKAUS, Huguette: Das Elsaß, das ich meine. Kleine Philosophie aus der Provinz. Straßburg 1998, S. 33-35. 
einem magischen Augenblick, denn Beten verscheuchte die bösen Geister ${ }^{8}$. Schutzengel-Darstellungen hingegen sind für das Wohnzimmer vorgesehen, da sie neben dem christlichen Inhalt gleichzeitig als Landschaftsbild einen adäquaten Wandschmuck für die Stubb bilden. Gebrauchsbilderbogen wie Tauferinnerungsblätter gaben mit ihren Texten ebenfalls eine Lebenshilfe für den Frommen.

Die künstlerische Quelle und die Art der Darstellung der Heiligenbilder interessieren die gläubigen Benutzer nicht. Stattdessen ist die Ikonographie der Heiligen so vertraut, daß sich ein Wechselspiel zwischen der Vorstellung vom Typus eines Heiligen und der Bestätigung durch das Bild ergibt und so das Gefühl der Richtigkeit der eigenen Imagination entsteht. Dies wiederum vermittelt die Sicherheit, im Kosmos der allgegenwärtigen biblischen Gestalten aufgehoben zu sein.

Daß DREIKAUS ihre knappen, aber präzisen Beschreibungen der Bilderbogen nicht gänzlich ihrem scharfen Erinnerungsvermögen verdankt, wird im Schlußteil des Kapitels deutlich. Denn dort verrät die Autorin an den Leser gewandt eher beiläufig, daß sie offenbar die genannten Bogen aktuell vorliegen hat: Machen Sie es wie ich. Sammeln Sie vor allem Heiligenbildchen'. Wie schon bei JEAN EGEN, werden hier Bilderbogen genutzt, um Wertvorstellungen aus der Kindheit mit Hilfe eines tradierten Bildmaterials zu transportieren. Bilder, die offensichtlich auch die nun erwachsenen Schriftsteller immer noch besitzen und schätzen.

Die Frage nach der Herkunft der Bilderbogen kann bei den genannten Beispielen nicht beantwortet werden. Aufgrund der geografischen Nähe könnten sie aus Épinal oder Wissembourg stammen: Für die genannten Autoren und wohl für die Mehrzahl der damaligen Bilderbogen-Benutzer ist diese Frage jedoch bedeutungslos. Wichtig allein ist, daß die Christusdarstellungen so waren, wie man sich eben Christusse vorstellte. An dieses Dogma hielten sich alle europäischen Bilderbogenhersteller schon aus kommerziellen Gründen, so daß zwischen den Verlagen ikonographische Abweichungen kaum und stilistische Unterschiede nur selten ausgeprägt vorkommen.

Die beiden Autoren beschreiben verschiedene Bilderbogen in Erinnerungen an Ereignisse aus der Jugendzeit. Obgleich damit das Alter der damaligen Rezipienten dem Klischee der schlichten und damit kindgerechten Bildform entspricht, verbirgt sich dahinter der erwachsene Betrachter, der im Nachhinein die prägende Bedeutung dieser Art von Bildvermittlung erkennt.

Ebd., S. 34.

Ebd., S. 36. 